\title{
Isoflurane preconditioning effects on brain damage induced by electromagnetic pulse radiation through epigenetic modification of BDNF gene transcription
}

\author{
Li-Ying Tian ${ }^{1 \#}$, Cheng-Kui Cai ${ }^{2 \#}$, Xia-Jing Zhang ${ }^{3}$, Xu-De Sun ${ }^{1}$ \\ ${ }^{1}$ Department of Anesthesiology, The Second Affiliated Hospital of Air Force Medical University, Xi'an, China; ${ }^{2}$ Department of Orthopedics, The \\ Second Affiliated Hospital of Shaanxi University of Chinese Medicine, Xianyang, China; ${ }^{3}$ Department of Anesthesiology, Xi'an No.4 Hospital, \\ Xi'an, China \\ Contributions: (I) Conception and design: XD Sun; (II) Administrative support: XD Sun; (III) Provision of study materials or patients: LY Tian, XJ \\ Zhang; (IV) Collection and assembly of data: CK Cai; (V) Data analysis and interpretation: LY Tian, CK Cai; (VI) Manuscript writing: LY Tian; (VII) \\ Final approval of manuscript: All authors. \\ "These authors contributed equally to this work. \\ Correspondence to: Xu-De Sun. Department of Anesthesiology, The Second Affiliated Hospital of Air Force Medical University, Xi'an 710038, China. \\ Email: 364546053@qq.com.
}

Background: The effects of electromagnetic pulse (EMP) radiation on cognitive impairment have attracted much attention, but the mechanism is still unclear. Regulation of brain-derived neurotrophic factor (BDNF) gene expression has been found to promote memory formation and neuronal survival. Isoflurane preconditioning (IP) was reported to have a neuroprotective effect. In this study, we verified the protective effect of IP against brain injury induced by EMP exposure and examined the relation of this effect with BDNF gene regulation.

Methods: Twenty-four hours before EMP exposure, rats were pretreated with $2 \%$ inhaled isoflurane for 30 minutes. At 24 hours after EMP injury, the Morris water maze test was carried out. Meanwhile, the other rats were executed and their brain tissues were used for Nissl staining, qRT-PCR, western blot and chromatin immunoprecipitation.

Results: The Morris water maze results showed that 2\% IP improved the spatial learning and memory ability of the rats. The Nissl staining results showed $2 \%$ of IP alleviated neuronal damage. Also, we detected the mRNA and protein expression of BDNF, and 2\% IP significantly increased the expression of BDNF. We also found the expression level of histone deacetylase 2 (HDAC2) was increased and that EMP exposure significantly decreased $\mathrm{H} 3$ acetylation, while $2 \%$ IP reversed these phenomena, individually, BDNF transcription was activated, and neurogenesis after EMP exposure was alleviated.

Conclusions: Our results suggested that $2 \%$ of IP alleviates cognitive impairment induced by EMP exposure in rats. Also, the sustained elevated level of BDNF gene transcription may be an essential mechanism for stimulating neurogenesis because of the increased level of HDAC2-dependent H3 acetylation.

Keywords: Electromagnetic pulse (EMP); neurogenesis; brain-derived neurotrophic factor (BDNF); histone acetylation; histone deacetylase 2 (HDAC2); isoflurane preconditioning

Submitted Aug 05, 2020. Accepted for publication Sep 11, 2020.

doi: 10.21037/apm-20-1655

View this article at: http://dx.doi.org/10.21037/apm-20-1655 


\section{Introduction}

With technological development, electromagnetic radiation has gained increasing attention as a unique kind of pollution. Many studies on the biological effects of electromagnetic radiation have shown that electromagnetic radiation affects all systems of the body $(1,2)$, including the central nervous system (CNS), gastrointestinal system, hematological system and reproductive system (3). The CNS is susceptible to electromagnetic pulse (EMP) radiation, which can cause learning and memory dysfunction (4). Earlier research found that a single $50 \mathrm{kV} / \mathrm{m}$ EMP can increase the expression of $A \beta$ in the hippocampus, resulting in impairment of learning and memory abilities (5). However, the mechanism underlying this effect is not yet precise. Therefore, the determination of the biological effects and mechanisms of EMP-induced cognitive impairment and the identification of effective medical measures to protect against the effects of EMP exposure are critical issues for clinical medicine.

Epigenetic mechanisms regulate gene expression without altering the primary DNA sequence (6-8). It participates in synaptic plasticity, learning, and memory $(9,10)$. Brainderived neurotrophic factor (BDNF) is the most abundant neurotrophic factor in the human body. It plays a vital role in neuronal development, synaptogenesis, learning, and memory $(11,12)$. Epigenetic changes are significant for long-term memory storage; elimination of acetyl groups from lysines on histone tails can inhibit histone deacetylase (HDAC), enhance histone acetylation and increase the gene expression of c-Fos and BDNF, enhancing new memory formation $(13,14)$. Other studies have shown $\mathrm{A} \beta$ activates microglia, triggers inflammatory responses, and further enhances the activity of HDAC2, resulting in changes in the epigenetic modification of BDNF and aggravating memory impairment $(15,16)$.

Few effective drugs are available to protect against the CNS damage caused by EMP exposure. Inhaled anesthetics have drawn increasing attention because of their neuroprotective effects. Isoflurane is a universally used inhaled anesthetic in the clinic. Some research has found isoflurane preconditioning (IP) can enhance tolerance of ischemia and play a protective role (17-19). Sun found IP supplied neuroprotection by directly regulating TLR4 expression, alleviating microglial activation, and neuroinflammation (20). Li confirmed that $2 \%$ of IP improved short-term and long-term neurologic outcome scores after cerebral ischemia and reduced the number of apoptotic cells. It is hypothesized that these effects of IP may be related to the level of the antiapoptotic protein Bcl-2 (21). Deng found that inhaled sevoflurane regulates the expression of apoptosis-related proteins and reduces neuronal damage caused by EMP exposure (4). Although IP can alleviate brain injury and supply neuroprotection during cerebral ischemia, it is not clear whether isoflurane also protects against EMP-induced brain injury.

Therefore, this study investigated the effects of IP on EMP-induced brain damage. The mechanism for the effects of IP was hypothesized to be related to epigenetic modification of BDNF gene transcription.

We present the following article in accordance with the ARRIVE reporting checklist (available at http://dx.doi. org/10.21037/apm-20-1655).

\section{Methods}

\section{Animals}

The Ethics Review Committee approved this study of Air Force Military Medical University, Xi'an, Shaanxi, China. One hundred Specific pathogen-free adult (3-month-old) male Sprague-Dawley (SD) rats (mean weight, $200 \pm 20 \mathrm{~g}$ ) were provided by the experimental animal center of Air Force Military Medical University. These animals are placed in free access to sterile laboratory particles and water, specific pathogen-free environments, and they were fed without further contact with EMP. SD rats were randomly divided into four groups (six rats per group): the control group (CON group), the EMP radiation group (EMP group), the IP group, and the IP + EMP group. These rats received IP inhaled $2 \%$ isoflurane for 30 minutes before EMP exposure. The experimental program was carried out according to the guidelines for animal experiments of the Air Force Military Medical University (Xi'an, China).

\section{EMP radiation model}

The instrument used in this experiment was developed jointly by the Air Force Military Medical University and Southwestern University according to international standards. The parameters used in this experiment were $400 \mathrm{kV} / \mathrm{m}$, the repetition frequency was $1 \mathrm{~Hz}$, the pulse width was $14 \mathrm{~ns}$, the rising time was $3.5 \mathrm{~ns}$, and the number of pulses was 400. EMP radiation exposure was conducted for 3 days. At 24 hours after EMP exposure, the rats were sacrificed, and brain sections were prepared for histology 
staining or Nissl staining.

\section{IP}

Rats that received IP were placed in a closed, circular Plexiglas box. The volatile anesthetic tank was connected to the inlet of the box, and the rats continuously inhaled 2\% isoflurane (batch number: H19980141, Hebei 9 Faction Pharmaceutical Co., Ltd.). The flow rate was $2 \mathrm{~L} /$ minutes; the outlet end was connected to the gas monitor, which continuously measured the gas concentration at the outlet side. A layer of soda lime (batch number: FA04-01, W. R. Grace S. A., USA) of suitable depth was placed at the bottom of the box to avoid inhalation of $\mathrm{CO}_{2}$. A disposable breathable napkin was used to isolate the rats from the soda-lime to prevent the ingestion or aspiration of sodalime dust particles. The anesthetic gas volatilization tank and oxygen flow meter were adjusted to support the concentration of isoflurane at the outlet of the gas port at $2 \%$, and the anesthetic was applied for 30 minutes. After this pretreatment and an interval of 24 hours, the model EMP injury was conducted on the rats.

\section{Morris water maze test}

At 24 hours after EMP injury, the Morris water maze test was used to detect the spatial learning and memory ability of the rats in each group. The test was performed in a 160 -cm-diameter circular tank with a black inner wall. The tank was divided into four quadrants. In one quadrant (target quadrant), a circular black platform with a diameter of $12 \mathrm{~cm}$ was placed $40 \mathrm{~cm}$ from the wall and $2 \mathrm{~cm}$ below the water surface. The ink was added to the water to hide the platform, and the water temperature was supported at $23 \pm 2{ }^{\circ} \mathrm{C}$. The entire experiment lasted 5 days, of which the navigation test lasted 4 days. Each animal was trained 4 times a day for 60 seconds per session, with 30 minutes intervals between each pair of training sessions. We randomly selected one quadrant as the entry point. We placed each rat in the pool at the wall and recorded the time to find the platform under the surface of the water. If the animal did not find the platform in 60 seconds, the animal was introduced to the platform for 10 seconds, and the escape latency was recorded as 60 seconds. Then, the spatial exploration test was conducted on the final test day. After the positioning navigation test, the platform was removed, and each rat was allowed to swim in the maze for 60 seconds. The time spent in the target quadrant over 60 seconds was recorded as an index of spatial memory in rats.

\section{Nissl staining}

Twenty-four hours after EMP exposure, the rats were anesthetized deeply with $2 \%$ pentobarbital sodium $(40 \mathrm{mg} / \mathrm{kg})$. After cardiac perfusion with $4 \%(\mathrm{w} / \mathrm{v})$ paraformaldehyde, the brain tissues of the hippocampal region were removed and placed in $4 \%$ (w/v) paraformaldehyde. The tissues were dehydrated in ethanol, embedded in paraffin blocks and sectioned at $5 \mathrm{~mm}$ per slice. The sections were processed for Nissl staining (22). Briefly, the sections were incubated for 40 minutes in tolridine blue, rinsed with distilled water, naturally dried at room temperature, dehydrated with an alcohol gradient, cleared with xylene, and mounted with neutral resin. Nissl staining (Beyotime Institute of Biotechnology, China) was observed as Nissl bodies found in the cytoplasm of surviving neurons. The integrated optical density/area of the staining in each group was acquired by 2 blinded investigators using Image-Pro Plus 5.1 software (Media Cybernetics, Inc., Bethesda, MD).

\section{Quantitative real-time PCR ( $q R T-P C R)$}

To assess the influence of IP on BDNF gene expression in the hippocampal tissues after EMP, we used qRT-PCR. Total RNA was extracted from the hippocampus, and cDNA was synthesized using AMV reverse transcriptase at $42{ }^{\circ} \mathrm{C}$ for 10 minutes, followed by incubation at $95^{\circ} \mathrm{C}$ for 2 minutes. ABI SYBR Green PCR Master Mix and the ABI StepOnePlus RealTime PCR System (CA, USA) were used. The reagents were incubated at $95^{\circ} \mathrm{C}$ for 2 minutes and then subjected to 45 cycles of $95^{\circ} \mathrm{C}$ for 5 seconds and $60^{\circ} \mathrm{C}$ for 10 seconds. The BDNF exon primers were $5^{\prime}$-TGAGCCGAGCTCATCTTTGC-3' (forward) and 5'-ATAGCGGGCGTTTCCTGAAG-3' (reverse). The primers for GAPDH were 5'-GGCAAGTTCAACGGCACAGT-3' (forward) and 5'ATGACATACTCAGCACCGGC-3' (reverse). The relative quantity of real-time PCR products was analyzed using an $\mathrm{ABI}$ 7900 HT system.

\section{Western blot analysis}

Western blot was used to analyze the expression of HDAC2, BDNF, and acetylated histone $\mathrm{H} 3$ in the hippocampus, as described previously. In summary, rats were deeply anesthetized and decapitated, and their brains were 

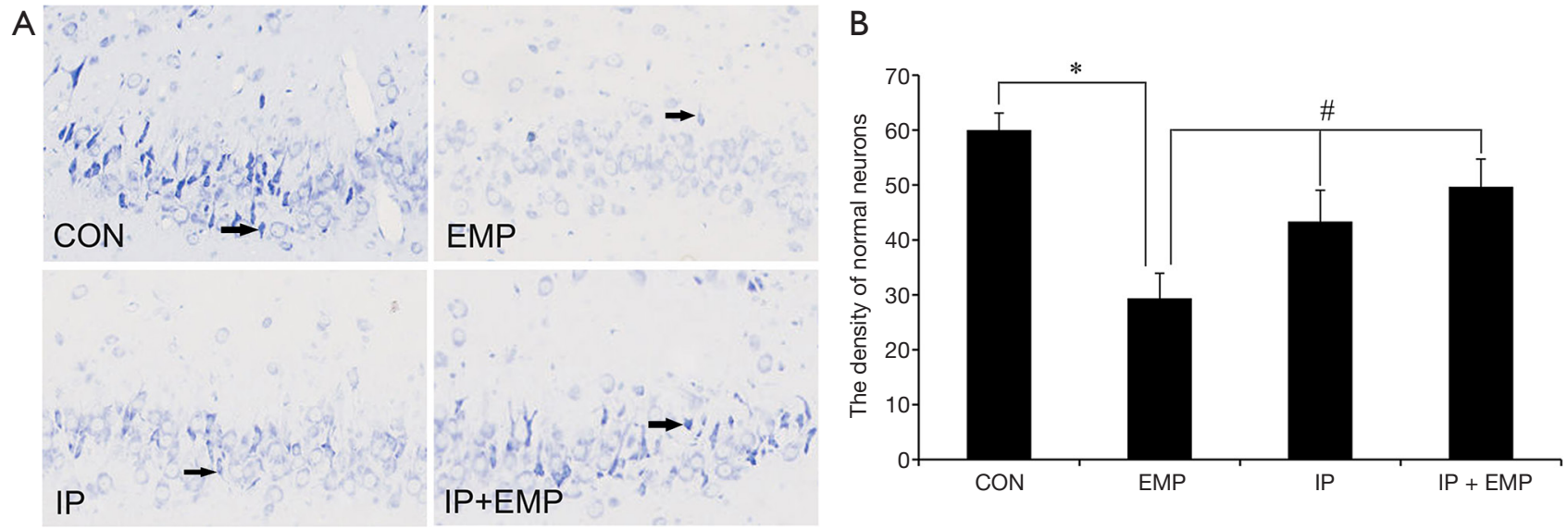

Figure 1 Effect of IP on neuronal damage in rat hippocampal region after EMP irradiation. (A) Nissl staining of neuronal damage in rat hippocampal area in 24 hours after EMP irradiation (400x); (B) the density of surviving neurons in rat hippocampal area in 24 hours after EMP irradiation. The mean \pm SEM represents the data. *, \#, $\mathrm{P}<0.05$. IP, isoflurane preconditioning; EMP, electromagnetic pulse.

removed and quickly placed on ice. Total protein from each hippocampus was acquired using an extraction kit (Beyotime, Shanghai, China) on ice. The total protein concentration of hippocampal samples was quantified with a BCA kit (Sigma, CA, USA). The following primary antibodies were used at the specified dilutions: rabbit anti-BDNF (1:1,000, Abcam), rabbit anti-HDAC2 (1:2,000, Abcam), rabbit anti-acetylated histone H3 (1:5,000, Millipore), and mouse anti-GAPDH $(1: 5,000$, Thermo). Secondary horseradish peroxidase (HRP)-conjugated goat anti-rabbit, or anti-mouse antibody (1:5,000, Pierce Biotechnology, Inc.) was used. The signals were detected using an ECL kit (Pierce) according to the manufacturer's instructions. The changes in relative protein expression are presented as the ratio of the integrated optical density of the target protein bands to the GAPDH protein band.

\section{Cbromatin immunoprecipitation (ChIP)}

The brain of rats was fixed with 4\% PFA solution and stored at $80{ }^{\circ} \mathrm{C}$ for ChIP. At room temperature, $1 / 10$ volumes of fresh $11 \%$ formaldehyde solution cross-linked chemically in the brain were homogenized and suspended for 15 minutes. The buffer cells were dissolved and crosslinked by dissolution and shearing of DNA. In this paper, we use Misonix Sonicator 3000 to treat the samples with $10 \times 30$ seconds pulse (pulse interval 90 seconds) at $4{ }^{\circ} \mathrm{C}$ at a power of 7 and then soak the samples in an ice bath. The whole-cell extract was incubated at $4{ }^{\circ} \mathrm{C}$ with $10 \mu \mathrm{g}$ antibody pre-incubated dynamic protein $\mathrm{G}$ beads for one night at
$4{ }^{\circ} \mathrm{C}$. Rinse with RIPA buffer washed 5 times and washed with TE containing $50 \mathrm{~mm} \mathrm{NaCl}$ once. The binding complex was eluted from the beads by heating at $65^{\circ} \mathrm{C}$ and occasionally by eddy current and cross-linking through nighttime culture at $65{ }^{\circ} \mathrm{C}$. Immunoprecipitation reaction DNA and DNA extracted from whole cells were purified by RNaseA therapy, protease $\mathrm{K}$, and polyphenol: chloroform: isoamyl alcohol extract. The purified DNA samples were normalized and analyzed by PCR. Anti-HDAC2 (\#12169) and anti-AcH3 (\#06-599) were used to the pulling down. The restored chromatin fragment was subjected to realtime PCR 32-40 cycles of 150-250 bp using primer pairs corresponding to specific mouse gene promoter regions.

\section{Statistical analysis}

In our study, we used SPSS22.0 software (SPSS, Chicago, IL) to analyze statistical data. The results are expressed as the means \pm standard error and were analyzed by one-way analysis of variance (ANOVA). $\mathrm{P}<0.05$ was used to show a statistically significant difference.

\section{Results}

IP alleviated neuronal damage in rats after EMP exposure

Nissl staining showed neuronal injuries, including cell loss, cellular swelling, nuclear pyknosis, and karyorrhexis, after EMP exposure. IP reduced these neuronal injuries (Figure $1 A$ ). The density of normal neurons was significantly 

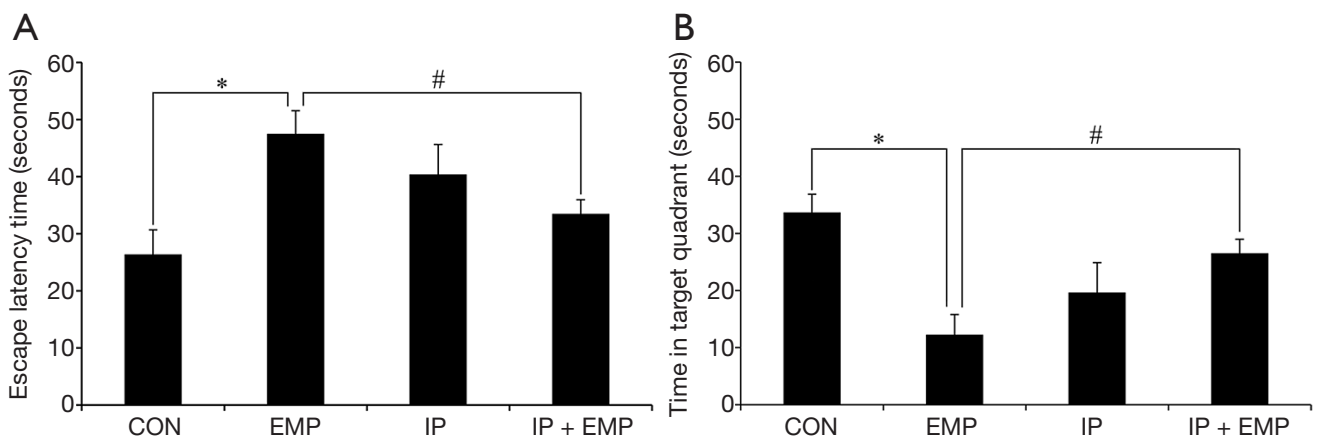

Figure 2 Effect of IP on neurobehavioral changes of EMP irradiated rats in the Morris water maze test. (A) The escape latency time in the Morris water maze test; (B) the activity time in the target quadrant in the Morris water maze test. *, ", $\mathrm{P}<0.05$. IP, isoflurane preconditioning; EMP, electromagnetic pulse.

lower in the EMP group than in the CON group (Figure $1 B, \mathrm{P}<0.05$ ), while the density of normal neurons in the IP and IP + EMP groups was significantly higher than that in the EMP group (Figure $1 B, \mathrm{P}<0.05$ ).

\section{IP improved spatial learning and memory ability in rats exposed to EMP radiation}

Compared with the CON group, the latency of escape station was prolonged (Figure $2 A, \mathrm{P}<0.05$ ) and the time of activity in the target quadrant was shortened in EMP group (Figure $2 B, \mathrm{P}<0.05$ ); however, the escape latency was shorter (Figure $2 A, \mathrm{P}<0.05$ ) and the activity time was longer in the target quadrant in the IP + EMP group than in the EMP group (Figure $2 B, \mathrm{P}<0.05$ ). These results showed that EMP radiation impaired the learning and memory ability of rats, while $2 \%$ of IP alleviated this learning and memory impairment.

\section{IP increased BDNF expression}

After EMP injury, we evaluated BDNF gene regulation and analyzed total BDNF gene expression by qRT-PCR and Western blot. The results showed that BDNF mRNA expression was decreased in the EMP group compared with the CON group (one-way ANOVA) (Figure $3 A, \mathrm{P}<0.05$ ). However, BDNF mRNA expression was higher in the IP + EMP group than in the EMP group (Figure $3 A, \mathrm{P}<0.05$ ). The Western blot results agreed with the qRT-PCR results, and verifying these findings (Figure $3 B, C, \mathrm{P}<0.05$ ). Our results showed that EMP inhibited BDNF expression, while $2 \%$ IP increased BDNF expression. This finding clarified that BDNF transcription is associated with the neuronal injury induced by EMP radiation and that 2\% IP alleviated this damage.

\section{IP alleviated reduced $\mathrm{H} 3$ acetylation after EMP through an HDAC2-related mechanism}

Next, we verified whether changes in histone acetylation can lead to EMP-induced impairment of neurogenesis and whether IP can reverse this effect. After EMP, acetylated $\mathrm{H} 3$ levels were markedly lower in the EMP group than in the CON group (Figure $4 A, B, \mathrm{P}<0.05$ ), whereas acetylated $\mathrm{H} 3$ levels were significantly higher in the IP and IP + EMP groups than in the EMP group (Figure $4 A, B, \mathrm{P}<0.05$ ). We also detected HDAC2 expression in the hippocampus. HDAC2 expression was significantly higher in the EMP group than in the $\mathrm{CON}$ group (Figure $4 C, D, \mathrm{P}<0.05$ ), while HDAC2 expression was lower in the IP and IP + EMP groups than in the EMP group (Figure $4 C, D, \mathrm{P}<0.05$ ). These results showed that IP alleviated neural injury by decreasing $\mathrm{H} 3$ acetylation after EMP through an HDAC2related mechanism.

The transcription of the BDNF gene is modified by epigenetic modification by a series of initiators. ChIP assays with RT-PCR were used to detect the level of acetylated histone in the promoter region of BDNF. After EMP, there was a significant reduction of $\mathrm{H} 3$ acetylation in the exon VI of the BDNF promoter (Kruskal-Wallis test, Figure 5, $\mathrm{P}<0.05$ ), which was consistent with the previously observed reduction of $\mathrm{BDNF}$ expression.

\section{Discussion}

In this research, the effects of EMP radiation on neuronal 

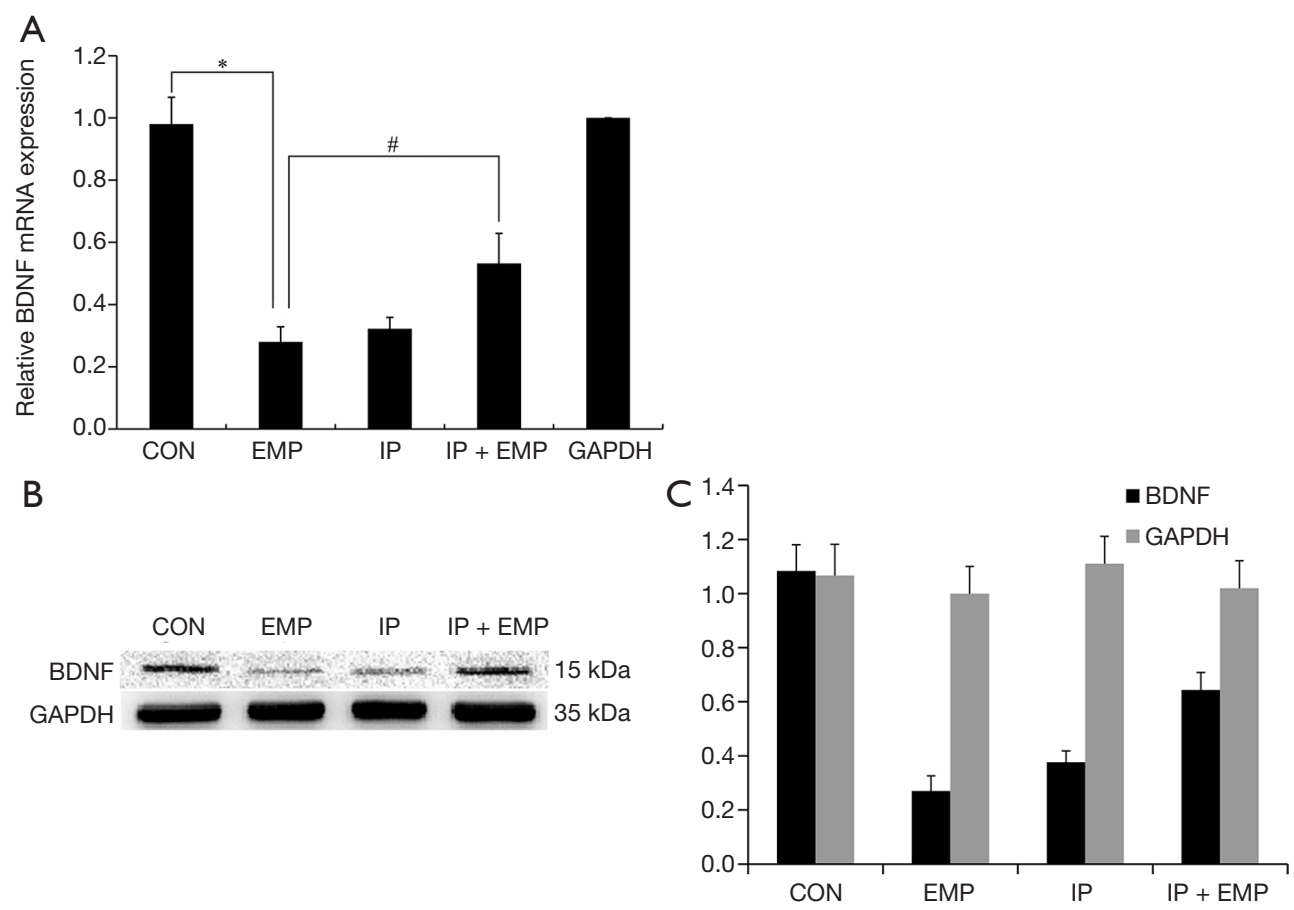

Figure 3 Expression of BDNF in hippocampal neurons after EMP irradiation. (A) Quantitative study on relative changes of BDNF expression; (B) representative western blot of BDNF at 24 hours after exposure to EMP radiation; (C) representative western blot of BDNF at 24 hours after exposure to EMP radiation. *, *, $\mathrm{P}<0.05$. The data are normalized to GADPH. IP, isoflurane preconditioning; EMP, electromagnetic pulse; BDNF, brain-derived neurotrophic factor.

A

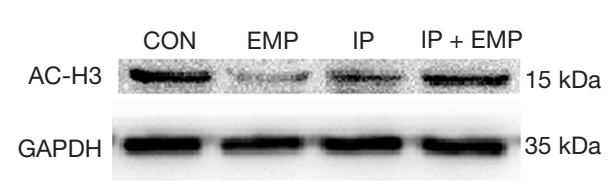

C

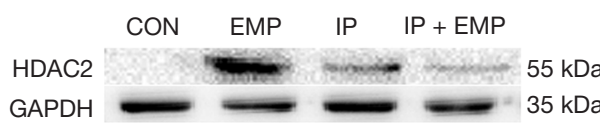

B

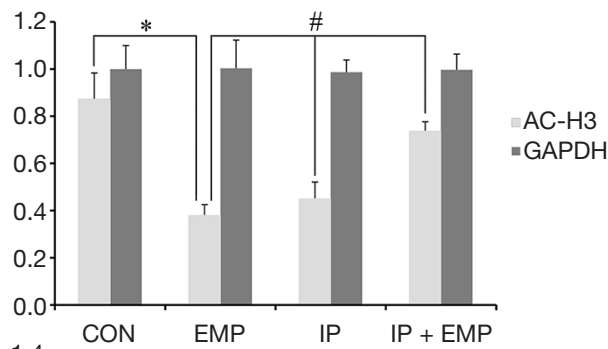

D

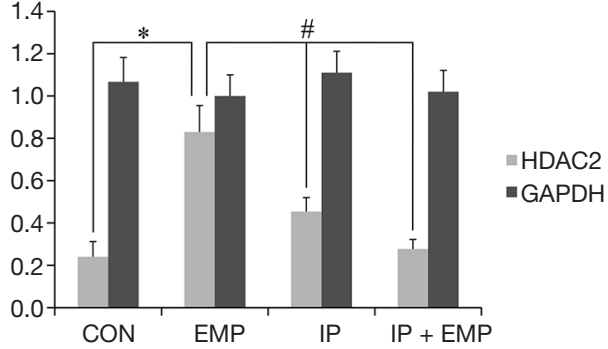

Figure 4 Expression of total H3 acetylation and HDAC2 in hippocampus neurons exposed to EMP radiation. (A) Representative western blot of total $\mathrm{H} 3$ acetylation at $24 \mathrm{~h}$ after exposure to EMP radiation; (B) quantitative study on relative changes of total H3 acetylation expression; (C) representative western blot of HDAC2 acetylation at $24 \mathrm{~h}$ after exposure to EMP radiation; (D) quantitative study on relative changes of HDAC2 expression. *, * $\mathrm{P}<0.05$. Data are presented as the mean \pm SEM. IP, isoflurane preconditioning; EMP, electromagnetic pulse; HDAC2, histone deacetylase 2. 


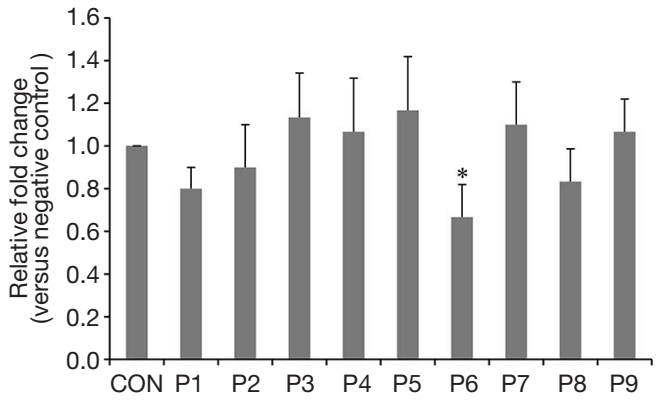

Figure 5 Chromatin immunoprecipitation (ChIP) and q-PCR analysis of $\mathrm{H} 3$ acetylation on brain-derived neurotrophic factor (BDNF) promoter regions. The level of acetylated $\mathrm{H} 3$ on BDNF promoter VI after electromagnetic pulse (EMP) irradiation was significantly decreased in rats compared with $\mathrm{CON}$. *, $\mathrm{P}<0.05$. The mean \pm SEM represents the data.

damage and cognitive impairment in rats were observed using EMP exposure of $400 \mathrm{kV} / \mathrm{m}, 400$ pulses, and $1 \mathrm{~Hz}$ for 3 consecutive days. Pretreatment with $2 \%$ inhaled isoflurane reduced the neuronal damage and cognitive impairment caused by EMP exposure. Further mechanistic studies revealed that the levels of BDNF expression and $\mathrm{H} 3$ acetylation were downregulated and that the level of HDAC2 expression was increased after EMP exposure. However, $2 \%$ of IP reversed these phenomena. These results implied that $2 \%$ of IP has protective effects against neuronal damage and cognitive impairment induced by EMP radiation. The mechanism underlying the effect of IP may be related to epigenetic modification of the BDNF gene.

With the rapid development of modern communication, the intensity of electromagnetic fields (EMFs) in the environment is increasing, and their scope is expanding. This development increases the possibility of human exposure to EMFs and will have side effects on humans (23). EMP radiation is a special EMF. An EMP is a short, highvoltage pulse characterized by spectral bandwidth ranging from $0 \mathrm{~Hz}$ to $1.5 \mathrm{GHz}(24,25)$. The nervous system is one of the most sensitive target organs for EMP exposure, and EMP exposure can change the permeability of the blood-brain barrier $(24,26)$, induce the activation of microglia in the brain (27), and even affect learning and memory ability in rats (4). In the present study, we studied hippocampal injury and neurocognitive impairment at 24 hours after EMP exposure. We first revealed EMP caused morphological injury to hippocampal neurons, as proved by Nissl staining. After EMP exposure, the Nissl body size was smaller, and the number of Nissl bodies was reduced, reflecting pathological damage.

We observed learning and memory deficits after EMP exposure. In the present study, we found that EMP exposure caused hippocampal neuronal injury and neurocognitive impairment. These results agree with earlier experimental results.

Many proteins nourish and protect brain neurons. BDNF, one of the essential neurotrophic factors, can repair or regenerate central and peripheral nerves (28). Many studies have shown that downregulation of BDNF in different regions of the brain is closely related to the occurrence of various neuropsychiatric diseases (22) and that the concentration of $\mathrm{BDNF}$ in brain tissue of $\mathrm{AD}$ patients is significantly decreased $(29,30)$. BNDF controls the plasticity of hippocampal neurons in adulthood, regulates the survival and integration of neonatal hippocampal neurons, and interferes with learning and memory (31). Studies have shown that the expression level of BDNF in serum is negatively correlated with memory function and visual acuity in $\mathrm{AD}$ patients (32). One study found that enhanced expression of BDNF improved memory formation and neuronal survival in neurological and psychiatric models (22). The results showed that the protein and mRNA expression of BDNF in the hippocampus of rats was significantly downregulated after EMP, suggesting that $\mathrm{BDNF}$ is related to the occurrence of neuropathies.

Epigenetic mechanisms, including DNA methylation, histone modifications, and noncoding RNA functions, are essential for the regulation of BDNF expression $(33,34)$. Many studies have shown that in some nervous system diseases, transcription factors can significantly induce chromatin remodeling, potentially through epigenetic modifications of the BDNF gene promoter region and subsequent regulation of hippocampal synaptic development and cognitive function $(35,36)$. Recent research has found that changes in the levels of acH3K9 and HDAC2 affect BDNF expression at both the transcript and protein levels (37). In our study, we verified that after EMP exposure, total $\mathrm{H} 3$ acetylation declined; however, the mRNA and protein expression of BDNF increased. We also found HDAC2 expression was increased and that BDNF levels were decreased after EMP exposure. Our experimental results agree with earlier research. Therefore, we confirmed that the level of acetylated $\mathrm{H} 3$ in BDNF promoter regions and the level of HDAC2 regulated the expression of BDNF. 
Isoflurane is a common halogenated ether used as a volatile inhaled anesthetic in the clinic. It has the following characteristics, low blood gas distribution coefficient, low airway irritation, and low cost. The CNS has a wide distribution of isoflurane targets, including the cerebral cortex, diencephalon, brainstem, and spinal cord. Injury (e.g., cerebral ischemia-reperfusion injury, cerebral hypoxic injury, or subarachnoid hemorrhage) has satisfactory neuroprotective effects. Also, its neuroprotective mechanism in practice mainly involves antiapoptotic and anti-inflammatory activities (38-40). Many mechanisms are underlying the neuroprotective effects of IP. Yan et al. suggested that isoflurane pretreatment can attenuate injury via ischemic preconditioning by activating the expression of HIF- $1 \alpha$ to up-regulate R/s6K of the Akt/ mT0R/s6K pathway (19). In Wang and Kong's study, the neuroprotective effect of IP against spinal cord ischemiareperfusion injury was related to activation of TREK1 expression (18). It was found that the neuroprotective effect of IP was related to the inhibitory effect of isoflurane on inducible nitric oxide synthase activity induced by ischemia and hypoxia (41). In our study, we used $2 \%$ isoflurane to pretreat the rats for 30 minutes before EMP exposure. We found $2 \%$ IP reduced neuronal injury, as proved by the increased volume of Nissl bodies, and the increased number of Nissl bodies, the reduction in pathological damage.

The cognitive ability of EMP-exposed rats was improved by $2 \%$ IP. Therefore, we inferred that the protective effect of IP against EMP exposure-induced damage might be related to the neuroprotective effect of isoflurane. We further studied the mechanism by which IP protects against EMP exposure-induced damage. We found 2\% IP up-regulated the level of acetylated $\mathrm{H} 3$, decreased the expression of HDAC2, and increased the mRNA and protein expression of BDNF, thus reducing hippocampal neuronal injury. Therefore, we inferred that the effect of $2 \%$ IP in reducing the damage caused by EMP exposure might be related to epigenetic modification of the BDNF gene.

Our previous research results show that the IP can relieve inflammation reaction caused by EMP through TLR4/ NFкB signaling pathways (42). Inflammation can cause the epigenetic changes, thus increasing the expression of HDAC2, reduce the expression of BDNF, cause brain damage, and IP can reverse this phenomenon.

There are some limitations in this study. Firstly, many other signaling pathways or genes may be involved in neuronal injury. It is not clear how these factors work and whether they interact with BDNF. Second, we observed the current study to assess neuronal injury, changes that may occur in epigenetic gene expression following EMP, and to detect associations between these two events. Hence, in our study, we established a brain injury model by EMP, and then used the model to instruction that epigenetic regulation of BDNF played a key role in the development of neuronal injury. Other studies need further validation of our conclusion, we plan to evaluate isoflurane post-processing effect on brain injury induced by EMP.

In summary, we found that EMP exposure can cause neuronal injury and cognitive damage in the hippocampus but that $2 \%$ IP can reduce neuronal injury and cognitive impairment induced by EMP exposure. The mechanism for the effect of IP may be related to the reduction of HDAC2 expression, the regulation of BDNF gene transcription, and the enhancement of BDNF expression.

\section{Acknowledgments}

Funding: This study was supported by the National Natural Science Foundation of China under Grant No. 3157040065.

\section{Footnote}

Reporting Checklist: The authors have completed the ARRIVE reporting checklist. Available at http://dx.doi. org/10.21037/apm-20-1655

Data Sharing Statement: Available at http://dx.doi. org/10.21037/apm-20-1655

Conflicts of Interest: All authors have completed the ICMJE uniform disclosure form (available at http://dx.doi. org/10.21037/apm-20-1655). The authors have no conflicts of interest to declare.

Ethical Statement: The authors are accountable for all aspects of the work in ensuring that questions related to the accuracy or integrity of any part of the work are appropriately investigated and resolved. The Ethics Review Committee approved this study of Air Force Military Medical University, Xi'an, Shaanxi, China. The experimental program was carried out according to the guidelines for animal experiments of the Air Force Military Medical University (Xi'an, China).

Open Access Statement: This is an Open Access article distributed in accordance with the Creative Commons Attribution- 
NonCommercial-NoDerivs 4.0 International License (CC BY-NC-ND 4.0), which permits the non-commercial replication and distribution of the article with the strict proviso that no changes or edits are made and the original work is properly cited (including links to both the formal publication through the relevant DOI and the license). See: https:// creativecommons.org/licenses/by-nc-nd/4.0/.

\section{References}

1. Kim JH, Lee JK, Kim HG, et al. Possible Effects of Radiofrequency Electromagnetic Field Exposure on Central Nerve System. Biomol Ther (Seoul) 2019;27:265-75.

2. He GL, Luo Z, Shen TT, et al. TREM2 Regulates Heat Acclimation-Induced Microglial M2 Polarization Involving the PI3K-Akt Pathway Following EMF Exposure. Front Cell Neurosci 2020;13:591.

3. Zuo WQ, Hu YJ, Yang Y, et al. Sensitivity of spiral ganglion neurons to damage caused by mobile phone electromagnetic radiation will increase in lipopolysaccharide-induced inflammation in vitro model. J Neuroinflammation 2015;12:105.

4. Deng B, Xu H, Zhang J, et al. Neuroprtective effects of sevofluraine against electromagnetic pulse-induced brain injury through inhibition of neuronal oxidative stress and apoptosis. PLoS One 2014;9:e91019.

5. Jiang DP, Li J, Zhang J, et al. Electromagnetic pulse exposure induces overexpression of Beta amyloid protein in rats. Arch Med Res 2013;44:178-84.

6. Blanco Rodríguez J, Camprubí Sánchez C. Epigenetic Transgenerational Inheritance. Adv Exp Med Biol 2019;1166:57-74.

7. Medici V, LaSalle JM. Genetics and epigenetic factors of Wilson disease. Ann Transl Med 2019;7:S58.

8. Stoccoro A, Coppede F. Role of Epigenetics in Alzheimer's Disease Pathogenesis. Neurodegener Dis Manag 2018;8:181-93.

9. Géranton SM, Fratto V, Tochiki KK, et al. Descending serotonergic controls regulate inflammation-induced mechanical sensitivity and methyl-CpG-binding protein 2 phosphorylation in the rat superficial dorsal horn. Molecular Pain 2008;4:35.

10. Géranton SM, Morenilla-Palao C, Hunt SP. A Role for Transcriptional Repressor Methyl-CpG-Binding Protein 2 and Plasticity-Related Gene Serum- and GlucocorticoidInducible Kinase 1 in the Induction of Inflammatory Pain States. J Neurosci 2007;27:6163-73.

11. Minichiello L. TrkB signalling pathways in LTP and learning. Nat Rev Neurosci 2009;10:850-60.

12. Park H, Poo MM. Neurotrophin regulation of neural circuit development and function. Nat Rev Neurosci 2013;14:7-23.

13. Lubin FD, Roth TL, Sweatt JD. Epigenetic regulation of BDNF gene transcription in the consolidation of fear memory. J Neurosci 2008;28:10576-86.

14. Stafford JM, Lattal KM. Is an epigenetic switch the key to persistent extinction? Neurobiol Learn Mem 2011;96:35-40.

15. Bie B, Wu J, Yang H, et al. Epigenetic suppression of neuroligin 1 underlies amyloid-induced memory deficiency. Nat Neurosci 2014;17:223-31.

16. Wang B Y, Zhong Y, Zhao Z, et al. Epigenetic suppression of hippocampal BDNF mediates the memory deficiency induced by amyloid fibrils. Pharmacol Biochem Behav 2014;126:83-9.

17. Cai M, Yang Q, Li G, et al. Activation of cannabinoid receptor 1 is involved in protection against mitochondrial dysfunction and cerebral ischaemic tolerance induced by isoflurane preconditioning. Br J Anaesth 2017;119:1213-23.

18. Wang $\mathrm{K}$, Kong X. Isoflurane preconditioning induces neuroprotection by up-regulation of TREK1 in a rat model of spinal cord ischemic injury. Biomol Ther (Seoul) 2016;24:495-500.

19. Yan W, Chen Z, Chen J, et al. Isoflurane preconditioning protects rat brain from ischemia reperfusion injury via upregulating the HIF-1 $\alpha$ expression through Akt/mTOR/ s6K activation. Cell Mol Biol 2016;62:38-44.

20. Sun M, Deng B, Zhao XY, et al. Isoflurane preconditioning provides neuroprotection against stroke by regulating the expression of the TLR4 signalling pathway to alleviate microglial activation. Sci Rep 2015;5:11445.

21. Li L, Zuo Z. Isoflurane preconditioning improves shortterm and long-term neurological outcome after focal brain ischemia in adult rats. Neuroscience 2009;164:497-506.

22. Nagahara AH, Tuszynski MH. Potential therapeutic uses of BDNF in neurological and psychiatric disorders. Nat Rev Drug Discov 2011;10:209-19.

23. Jiang DP, Li JH, Zhang J, et al. Long-term electromagnetic pulse exposure induces Abeta deposition and cognitive dysfunction through oxidative stressand over expression of APP and BACE1. Brain Res 2016;1642:10-9.

24. Zhou JX, Ding GR, Zhang J, et al. Detrimental effect of electromagnetic pulse exposure on permeability of in vitro blood brain-barriermodel. Biomed Environ Sci 2013;26:128-37.

25. Qiu LB, Ding GR, Li KC, et al. The role of protein 
kinase $\mathrm{C}$ in the opening of blood-brain barrier induced by electromagnetic pulse. Toxicology 2010;273:29-34.

26. Zhou Y, Qiu LB, An GZ, et al. Effects of electromagnetic pulse exposure on gelatinase of blood-brain barrier in vitro. Electromagn Biol Med 2017;36:1-7.

27. Yang LL, Zhou Y, Tian WD, et al. Electromagnetic pulse activated brain microglia via the p38 MAPK pathway. Neurotoxicology 2016;52:144-9.

28. Hyman C, Hofer M, Barde YA, et al. BDNF is a neurotrophic factor for dopaminergic neurons of the substantia nigra. Nature 1991;350:230-2.

29. Faria MC, Goncalves GS, Rocha NP, et al. Increased plasma levels of BDNF and inflammatory markers in Alzheimer's disease. J Psychiatr Res 2014;53:166-72.

30. Fahnestock M, Garzon D, Holsinger RM, et al. Neurotrophic factors and Alzheimer's disease: are we focusing on the wrong molecule? J Neural Transm Suppl 2002;(62):241-52.

31. Vilar M, Mira H. Regulation of Neurogenesis by Neurotrophins during Adulthood: Expected and Unexpected Roles. Front Neurosci 2016;10:26.

32. O'Bryant SE, Hobson VL, Hall JR, et al. Serum BrainDerived Neurotrophic Factor Levels Are Specifically Associated with Memory Performance among Alzheimer's Disease Cases. Dement Geriatr Cogn Disord 2011;31:31-6.

33. Mitchelmore C, Gede L. Brain derived neurotrophic factor: epigenetic regulation in psychiatric disorders. Brain Res 2014;1586:162-72.

34. Varendi K, Kumar A, Härma MA et al. miR-1, miR-10b, miR-155, and miR-191 are novel regulators of BDNF. Cell Mol Life Sci 2014;71:4443-56.

35. Dong E, Dzitoyeva SG, Matrisciano F, et al. Brain-derived neurotrophic factor epigenetic modifcations associated with schizophrenia-like phenotype induced by prenatal stress in mice. Biol Psychiatry 2015;77:589-96.

36. Santos AR, Comprido D, Duarte CB. Regulation of local translation at the synapse by BDNF. Prog Neurobiol 2010;92:505-16.

37. Jiang H, Zhang X, Lu J, et al. Antidepressant-Like Effects of Acupuncture-Insights From DNA Methylation and Histone Modifications of Brain-Derived Neurotrophic Factor. Front Psychiatry 2018;9:102.

38. Xiang HF, Cao DH, Yang YQ, et al. Isoflurane protects against injury caused by deprivation of Oxygen and glucose in microglia through regulation of the Toll-like receptor4 pathway. J Mol Neurosci 2014;54:664-70.

39. Yin J, Li H, Feng C, et al. Inhibition of brain ischemiacaused notch activation in microglia may contribute to isoflurane postconditioning-induced neuropretection in male rats. CNS Neurol Disord Drug Targets 2014;13:718-32.

40. Zhao P, Ji G, Xue H, et al. Isoflurane postconditioning improved long-term neurological outcome possibly via inhibiting the mitochondrial permeability transition pore in neonatal rats after brain hypoxia-ischemia. Neuroscience 2014;280:193-203.

41. Xie LK, Yang SH. Brain globins in physiology and pathology. Med Gas Res 2016;6:154-63.

42. Li JJ, Deng B, Zhang XJ, et al. Isoflurane Preconditioning Attenuates Brain Injury Induced by Electromagnetic Pulse via the TLR4/NFкB Signaling Pathway. Oxid Med Cell Longev 2019;2019:9653494.

(English Language Editor: J. Chapnick)
Cite this article as: Tian LY, Cai CK, Zhang XJ, Sun XD. Isoflurane preconditioning effects on brain damage induced by electromagnetic pulse radiation through epigenetic modification of BDNF gene transcription. Ann Palliat Med 2020;9(5):3418-3427. doi: 10.21037/apm-20-1655 